\title{
ON THE DIAMETERS OF COMPACT RIEMANN SURFACES
}

\author{
FWU-RANQ CHANG ${ }^{1}$
}

\begin{abstract}
We derive an inequality relating the diameter and the length of a simple closed geodesic on a compact Riemann surface.
\end{abstract}

1. Introduction. Let $S$ be a compact Riemann surface of genus $g \geqslant 2$. Let $G$ be the Fushsian group representing $S$. The metric on $S=U / G$ is the Poincaré metric, which is induced from the Poincaré metric $|d z| / y$ in the upper half plane $U$. This is the only metric we use throughout this paper. Let $\alpha$ be a simple closed geodesic on $S$. If we vary the conformal structure on $S$ so that the length of $\alpha$ goes to zero, then the surface $S$ goes to the boundary of Teichmüller space. This kind of deformation was justified by Keen [2] using the existence of collars on Riemann surfaces.

In [4] Mumford proved a general compactness theorem for Fuchsian groups of the first kind under the hypotheses that all groups $G$ considered are torsion free and $U / G$ is compact. These additional conditions were removed by Bers [1]. Along the lines of Mumford's proof, he derived an inequality relating the diameter and a shortest simple closed geodesic on a compact Riemannian manifold. In Riemann surface theory it can be read as follows. Let $S$ be a compact Riemann surface of genus $g$. Let $\mathrm{d}$ be the diameter of $S$ and let $\mathbf{m}$ be the length of a shortest simple closed (nontrivial) geodesic on $S$; then

$$
\text { md } \leqslant 2 \operatorname{area}(S) .
$$

In this paper, we shall find a sharper inequality and an inequality in the reverse direction, from which we conclude that if $S$ goes to the boundary of Teichmüller space, then the diameter of $S$ will go to infinity. As a matter of fact, the inequality we found is true for any simple closed geodesic (not necessarily of shortest length). Moreover, we allow the group $G$ representing $S$ to have elliptic elements.

The author wishes to thank Professor Bernard Maskit for many helpful suggestions.

2. Definitions and statements of results. Let $G$ be a finitely generated Fuchsian group of the first kind with signature $\sigma=\left(g, n ; \nu_{1}, \nu_{2}, \ldots, \nu_{n}\right)$, where $g$ and $n$ are positive integers or zero, the $\nu_{i}$ are integers or the symbol

Received by the editors May 18, 1976 and, in revised form, November 11, 1976.

AMS (MOS) subject classifications (1970). Primary 30A46.

'This paper is based on a part of the author's $\mathrm{Ph}$. D. dissertation written under Professor Bernard Maskit at the State University of New York, Stony Brook, 1976. 
$\infty$, and $2<\nu_{1} \leqslant \nu_{2} \leqslant \cdots \leqslant \nu_{n} \leqslant \infty$. It is known that the area of $G$ is given by

$$
\begin{aligned}
\mathbf{A} & =\operatorname{area}(G)=\iint_{U / G} y^{-2} d x d y \\
& =2 \pi\left(2 g-2+n-\nu_{1}^{-1}-\cdots-\nu_{1}^{-1}\right)>0 .
\end{aligned}
$$

Note that $U / G$ is compact if and only if $n=0$ or $\nu_{n}<\infty$; we call such signature of compact type. We denote by $\operatorname{diam}(G)$ the diameter of $U / G$. The diameter is finite if and only if $U / G$ is compact.

We denote by $X(\sigma)$ the set of conjugacy classes [G] of Fuchsian groups $G$ with signature $\sigma$. Mumford's compactness theorem states that if $\sigma$ is of compact type, then the subset of $X(\sigma)$ corresponding to groups $G$ so that all geodesics on $U / G$ have length $\geqslant c$ (a constant) is compact.

Let $S=U / G$ be a compact Riemann surface. Let $\alpha$ be a minimal geodesic of length a on $S$. By a band $B$ around $\alpha$ of radius $b$ we mean the union of all geodesics on $S$ perpendicular to $\alpha$, where each is of length $b$. Since the Poincaré metric on $U$ is given by

$$
\lambda(z)|d z|=2(z-\bar{z})^{-1}|d z|,
$$

the ray $\theta=\theta_{0}, 0<\theta_{0}<\pi / 2$, is of distance $\log \left|\csc \theta_{0}+\cot \theta_{0}\right|$ to the imaginary axis $\theta=\pi / 2$. Lifting $B$ up to the upper half plane $U$ with $\alpha$ lying on the imaginary axis, an easy computation shows that $B$ is a region bounded by the curves $\rho=1, \rho=e^{a}, \theta=\csc ^{-1}(\cosh (b))$ and $\theta=\pi-\csc ^{-1}(\cosh (b))$. One easily sees that the noneuclidean area of $B$ is $2 a \sinh (b)$.

Now we state the main results.

THEOREM. Let $G$ be a Fuchsian group so that $U / G$ is compact with $\mathbf{d}=\operatorname{diam}(G)$ and $\mathbf{A}=\operatorname{area}(G)$. Then we have

$$
2 \mathbf{r} \sinh (\mathbf{d})>\mathbf{A} \text {, }
$$

where $\mathbf{r}$ is the length of a simple closed geodesic on $S$; and

$$
2 \sinh (\mathbf{m} / 4) \mathbf{d} \leqslant \mathbf{A} \text {, }
$$

where $\mathbf{m}$ is the length of a shortest simple closed geodesic on $S$.

The following corollaries follow from the previous theorem together with Mumford's compactness theorem.

Corollary 1. Let $\sigma$ be the type $(g, 0)$. If $G$ is on the boundary of Teichmüller space $X(\sigma)$, then $U / G$ is not compact.

With Bers' generalization of Mumford's theorem, we have

Corollary 2 (Bers [1]). Let $\sigma$ be of compact type. The subset of $X(\sigma)$ corresponding to groups $G$ with $\operatorname{diam}(G) \leqslant c<\infty$ is compact.

3. Proof of the theorem. Let $\beta$ be a simple closed geodesic on $S=U / G$ with length $\mathbf{r}$. Let $p$ be any point on $\beta$ which is not a fixed point of $G$. Form 
the Dirichlet region $D$ of $G$ in $U$ with center at $p=i e^{r / 2}$ and $\beta$ lying on the imaginary axis of $U$. Then $D$ is contained in a strip bounded by the curves $\rho=1$ and $\rho=e^{r}$. We construct a band $B$ around $\beta$ of radius d. Lifting $B$ to $U, B$ is the region bounded by the curves $\rho=1, \rho=e^{r}, \theta=\csc ^{-1}(\cosh (d))$ and $\theta=\pi-\csc ^{-1}(\cosh (\mathrm{d}))$. Since any point $q$ on $S$ is of distance at most $\mathbf{d}$ to the center $p=i e^{\mathrm{r} / 2}, q$ is of distance at most $\mathrm{d}$ to $\beta$. Thus $D \subset B$ and hence,

$$
A=\operatorname{area}(G)=\operatorname{area}(D) \leqslant \operatorname{area}(B)=2 \mathrm{r} \sinh (\mathbf{d}) .
$$

This proves (1).

Let $\delta$ be a minimal geodesic realizing the diameter of $S$ with endpoints $x$ and $y$. Let $B^{\prime}$ be the band around $\delta$ of radius $\mathrm{m} / 4$. We first prove that no two such geodesics of $B^{\prime}$ meet. Suppose $\delta_{1}$ and $\delta_{2}$ meet at the point $w$. Let $z_{1}, z_{2}$ be the points on $\delta$ from which $\delta_{1}, \delta_{2}$ originate, and $e$ be the distance from $z_{1}$ to $z_{2}$ along $\delta$. Then we can go from $x$ to $y$ by going from $x$ to $z_{1}$ on $\delta$, following $\delta_{1}$, then $\delta_{2}$ and going from $z_{2}$ to $y$ on $\delta$. This has length $\leqslant \mathbf{d}-\mathbf{e}+$ $\mathrm{m} / 2$, and since $\delta$ is the shortest path from $x$ to $y, \mathbf{d}<\mathbf{d}-\mathbf{e}+\mathbf{m} / 2$, i.e., e $<\mathrm{m} / 2$. But then $\delta_{1}, \delta_{2}$ and the part of $\delta$ between $z_{1}$ and $z_{2}$ is a closed curve $\tau$ of length at most $\mathbf{m} . \tau$ is certainly not homotopic to zero, for otherwise $\tau$ would be lifted to a triangle in the upper half plane $U$ with two right interior angles. Moreover, $\tau$ has corners and so it is not a geodesic. Therefore there is a closed geodesic freely homotopic to $\tau$ of length $<\mathbf{m}$, which is impossible.

This shows that, with the choice of the radius $\mathrm{m} / 4$, the whole band $B^{\prime}$ is contained in $S$. So we have

$$
2 \sinh (\mathbf{m} / 4) \mathbf{d}=\operatorname{area}\left(B^{\prime}\right) \leqslant \operatorname{area}(S)=\mathbf{A},
$$

and the theorem is proved.

\section{REFERENCES}

1. L. Bers, A remark on Mumford's compactness theorem, Israel J. Math. 12 (1972), 400-407.

2. L. Keen, Collars on Riemann surfaces, Ann. of Math. Studies, No. 79, Princeton Univ. Press, Princeton, N. J., 1974, pp. 263-268.

3. J. Lehner, Discontinuous groups and automorphic functions, Math. Surveys, no. 8, Amer. Math. Soc., Providence, R. I., 1964.

4. D. Mumford, A remark on Mahler's compactness theorem, Proc. Amer. Math. Soc. 28 (1971), 289-294.

Department of Mathematics, State University of New York, Stony Brook, New York 11794

Current address: Department of Economics, University of Chicago, Chicago, Illinois 60637 